Reprod. Nutr. Dévelop., 1985, 25 (2), 379-388.

\title{
Effets de l'adrénaline et de la phényléphrine sur l'extraction du lait au cours de la traite mécanique de la vache
}

\author{
J. BERNABÉ, Marie-José RICORDEL
}

I.N.R.A., Laboratoire de Recherches sur la Traite,

65, rue de Saint-Brieuc, 35042 Rennes Cedex, France.

Summary. Effects of adrenaline and phenylephrine on milk extraction during mechanical milking of cows.

During mechanical milking in the morning between days 60 and 130 of lactation, 6 or 10 French Friesian Pie-Noire (FFPN) cows were given an intrajugular injection of $0.3 \mathrm{mg}$ of adrenaline or $5 \mathrm{mg}$ of phenylephrine (table 1). The treatments, each given 7 times to each cow, were alternated every other day with a control injection of $0.9 \% \mathrm{NaCl}$.

The adrenaline caused:

- a non-significant decrease in total milk yield ( $-4.9 \%: 11.57$ I vs 12.17 I with $\mathrm{NaCl}$ ) and in the volume of machine-stripped milk (0.360 I vs 0.430 I with $\mathrm{NaCl}$ ) ;

- an increase $(+8.7 \%)$ in milk flow $(1.165 \mathrm{l} / \mathrm{min}$ vs $1.072 \mathrm{I} / \mathrm{min}$ with $\mathrm{NaCl}$; table 4).

Phenylephrine caused a significant $10 \%$ reduction in total milk yield $(13.35$ I vs 14.84 with $\mathrm{NaCl})$ but had no effect on the volume of machine-stripped milk $(0.235$ I vs 0.295 I with $\mathrm{NaCl})$ or on milk flow $(0.961 \mathrm{I} / \mathrm{min}$ vs $0.962 \mathrm{I} / \mathrm{min}$ with $\mathrm{NaCl}$; table 3).

In agreement with our previous results (Bernabé and Peeters, 1980 ; Bernabé and Ricordel, 1985), $\beta$-receptor (adrenaline) stimulation appeared to increase milk flow by relaxing the teat muscles. However, when contact was avoided between the teats and the milking machine, the stimulation of teat $\alpha$-receptors (phenylephrine) did not inhibit milk flow. It is supposed that during milking the machine caused the teat tissue to contract enough to mask the effect of the $\alpha$-mimetic treatment on milk flow.

\section{Introduction.}

Avant la traite, il est fréquent d'observer chez la vache des échappements spontanés de lait qui peuvent, respectivement, être diminués ou augmentés par l'injection intra-artérielle de sympathomimétiques $\alpha$ et $\beta$ (Bernabé et Peeters, 1980).

Récemment, nous avons pu démontrer que l'administration intrajugulaire $\mathrm{d}^{\prime}$ isoprénaline ( $\beta$ mimétique) permettrait d'accroître significativement le débit lacté au cours de la traite sans affecter le volume de lait extrait avant et pendant l'égouttage (Bernabé et Ricordel, 1985). Inversement, il restait à vérifier si, une drogue sympathique stimulant sélectivement les $\alpha$ récepteurs, était susceptible de 
freiner ce débit. Nous avons donc choisi la phényléphrine qui induit à la fois une vasoconstriction et une hypertonicité du trayon. De plus, afin de mieux interpréter l'effet des sympathomimétiques sur l'écoulement du lait (et en particulier de dissocier leur influence respective sur la musculature lisse et sur le système artérioveineux du trayon), nous avons utilisé l'adrénaline qui, comme la phényléphrine, possède des propriétés vasoconstrictrices mais qui se différencie de cette dernière par ses effets de relaxation de l'organe.

\section{Matériel et méthodes.}

1) Présentation générale de l'essai. - L'étude des effets de la phényléphrine (PHE) est conduite sur 10 vaches FFPN entre le $63^{\mathrm{e}}$ jour et le $83^{\mathrm{e}}$ jour de lactation. Celle des effets de l'adrénaline (A) lui a été postérieure (entre le $110^{\mathrm{e}}$ et le $130^{\mathrm{e}}$ jour) et n'a été réalisée que sur 6 animaux seulement (tabl. 1).

Chacune des 2 drogues est administrée au minimum 7 fois à chaque vache en alternance journalière (samedi et dimanche exceptés) avec une solution témoin de $\mathrm{NaCl} 0,9 \%$. Les injections ont toujours lieu au cours de la traite mécanique du matin, légèrement retardée (1), grâce à un cathéter de polyéthylène (PE $90, B e c-$ ton et Dickinson) placé en permanence dans la veine jugulaire (cf. 2).

Les modifications éventuelles du débit du lait et des volumes extraits avant et pendant l'égouttage à la machine sont contrôlées à l'aide d'un système d'enregistrement automatique (cf. 3).

2) Préparation des drogues et méthode d'injection. - L'adrénaline (épinéphrine bitartrate : Sigma réf. E-4375) et la phényléphrine (Néosynéphrine ${ }^{\circledR}$ Badrial, $5 \mathrm{mg} / \mathrm{ml}$ injectable) sont diluées dans une solution de $\mathrm{NaCl} 0,9 \%$ respectivement additionnée ou non d'acide ascorbique $0,6 \mu \mathrm{g} / \mathrm{ml}$ de telle façon, qu'à chaque injection, $0,3 \mathrm{mg}$ de $\mathrm{A}$ ou $5 \mathrm{mg}$ de PHE soient toujours administrés dans un volume de $5 \mathrm{ml}{ }^{(2)}$. Ces $5 \mathrm{ml}$ sont immédiatement " poussés " par $3 \mathrm{ml}$ de $\mathrm{NaCl} 0,9 \%$ afin qu'aucun reliquat de drogue ne subsiste dans le cathéter.

Le moment d'injection se situe environ $70 \mathrm{~s}(\mathrm{~A})$ et $110 \mathrm{~s}$ (PHE) après la pose du de̊rnier gobelet-trayeur. Cette opération est réalisée dans les mêmes conditions que celles déjà décrites par Bernabé et Ricordel (1985).

3) Enregistrement de la production laitière et de la cinétique d'émission du lait. - La chaîne de mesure composée de 4 suiveurs de niveau Sefram SN 50 (Labussière et Martinet, 1964) et de 2 tables traçantes potentiométriques à double voie (JJ Lloyd $\mathrm{CR} 600$ ) est identique à celle que nous avons utilisée précédemment (Bernabé et Ricordel, 1985).

Les tracés permettent de définir pour chaque quartier : - le débit de pré injection (DO en $1 / \mathrm{min}$ ); - le débit postérieur à l'injection de $\mathrm{NaCl}$ (D) en

(1) Horaires normaux entre $7 \mathrm{~h} 30$ et $8 \mathrm{~h} 30$ repoussés pour l'expérimentation entre $9 \mathrm{~h} 00$ et $11 \mathrm{~h} 30$, les vaches étant traites une à une.

(2) II en est de même pour la solution témoin $(\mathrm{NaCl} 0,9 \%)$. 


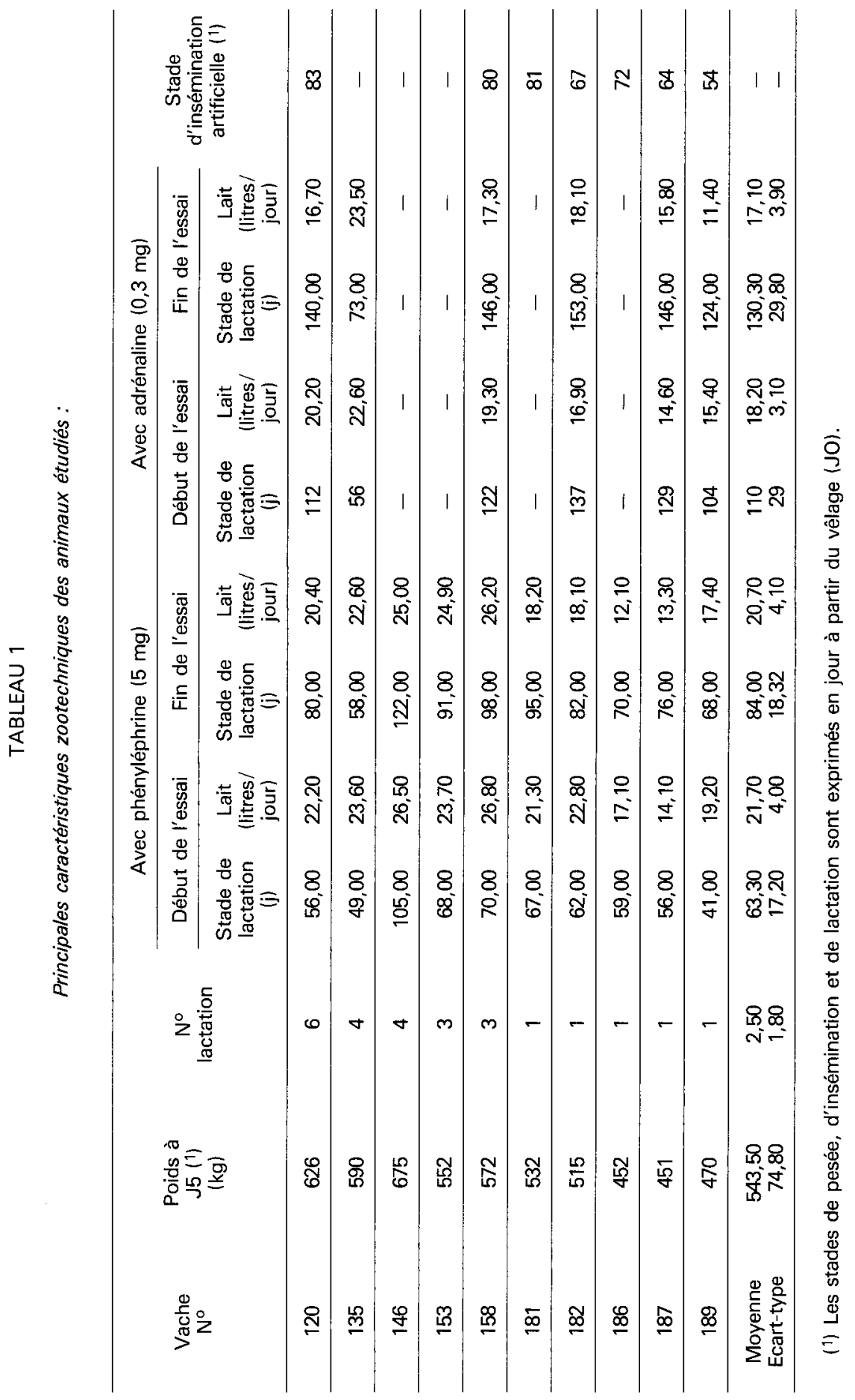


$\mathrm{l} / \mathrm{min}$ ); - le débit postérieur à l'injection du sympathomimétique (D2 en $\mathrm{l} / \mathrm{min}$ ) ; - le volume de lait d'égouttage (EM en I) ; - le volume de lait total (LT en I).

4) Conduite d'élevage et conditions de traite. - Les animaux sont logés, nourris et traits dans des conditions analogues à celles précédemment rapportées (Bernabé et Ricordel, 1985).

\section{Résultats.}

Effets sur la production totale de lait.

Avec la phényléphrine. - L'administration intrajugulaire de $5 \mathrm{mg}$ de PHE entraîne une réduction hautement significative $(-10,0 \%)$ de la production laitière moyenne du matin (14,84 I sous $\mathrm{NaCl}$ versus 13,35 I sous $\mathrm{PHE})$. Cet effet est constaté sur chacune des 10 vaches de l'essai et sur la quasi totalité de leurs quartiers (37 sur 40 ). II varie de $-2 \%$ (vache 153 ) à $-17 \%$ (vache 135 ) mais n'atteint le seuil de signification que chez $2 / 3$ des sujets (tabl. 2).

TABLEAU 2

Effets des injections de $\mathrm{NaCl}$, de PHE ET d'A sur les productions totales de lait de chaque quartier.

\begin{tabular}{|c|c|c|c|c|c|c|c|}
\hline $\begin{array}{l}\text { Vache } \\
N^{\circ}\end{array}$ & Quartier & $\begin{array}{c}\text { Lait total } \\
\text { produit sous } \\
\text { influence de } \\
\mathrm{NaCl} \mathrm{0,9 \%}\end{array}$ & $\begin{array}{l}\text { Lait total } \\
\text { produit sous } \\
\text { influence de } \\
\text { la PHE } 5 \mathrm{mg}\end{array}$ & $\begin{array}{l}\text { Effet de } \\
\text { la PHE } \\
\text { sur le lait } \\
\text { total }\end{array}$ & $\begin{array}{l}\text { Lait total } \\
\text { produit sous } \\
\text { influence de } \\
\mathrm{NaCl} 0,9 \%\end{array}$ & $\begin{array}{c}\text { Lait total } \\
\text { produit sous } \\
\text { influence de } \\
\text { I'A }\end{array}$ & $\begin{array}{l}\text { Effet de } \\
\text { l'A } \\
\text { sur le lait } \\
\text { total }\end{array}$ \\
\hline 120 & $\begin{array}{l}\text { P.D. } \\
\text { A.D. } \\
\text { A.G. } \\
\text { P.G. } \\
\text { Total }\end{array}$ & $\begin{array}{r}5,670 \\
3,360 \\
2,290 \\
4,990 \\
16,320\end{array}$ & $\begin{array}{r}5,290 \\
2,940 \\
2,310 \\
4,520 \\
15,020\end{array}$ & $\begin{array}{l}-0,380 \\
-0,420 \\
+0,020 \\
-0,470 \\
-1,300\end{array}$ & $\begin{array}{r}4,460 \\
2,380 \\
1,740 \\
3,740 \\
12,320\end{array}$ & $\begin{array}{r}4,650 \\
2,230 \\
1,710 \\
3,930 \\
12,520\end{array}$ & $\begin{array}{l}+0,190 \\
-0,150 \\
-0,030 \\
+0,190 \\
+0,200\end{array}$ \\
\hline 135 & $\begin{array}{l}\text { P.D. } \\
\text { A.D. } \\
\text { A.G. } \\
\text { P.G. } \\
\text { Total }\end{array}$ & $\begin{array}{r}6,030 \\
3,720 \\
2,450 \\
5,570 \\
17,770\end{array}$ & $\begin{array}{r}5,070 \\
2,970 \\
2,140 \\
4,560 \\
14,740\end{array}$ & $\begin{array}{l}-0,960^{* *} \\
-0,750^{* * *} \\
-0,310^{* *} \\
-1,010^{* * *} \\
-3,030^{* * *}\end{array}$ & $\begin{array}{r}6,370 \\
3,770 \\
2,430 \\
5,750 \\
18,320\end{array}$ & $\begin{array}{r}5,340 \\
3,190 \\
2,100 \\
4,860 \\
15,490\end{array}$ & $\begin{array}{l}-1,030^{* * *} \\
-0,580^{* * *} \\
-0,330^{* *} \\
-0,890^{* *} \\
-2,830^{* * *}\end{array}$ \\
\hline 146 & $\begin{array}{l}\text { P.D. } \\
\text { A.D. } \\
\text { A.G. } \\
\text { P.G. } \\
\text { Total }\end{array}$ & $\begin{array}{r}4,580 \\
4,570 \\
4,370 \\
4,630 \\
18,150\end{array}$ & $\begin{array}{r}4,090 \\
3,660 \\
3,680 \\
4,040 \\
15,480\end{array}$ & $\begin{array}{l}-0,490^{*} \\
-0,910^{* * *} \\
-0,690^{* * *} \\
-0,590^{* * *} \\
-2,670^{* * *}\end{array}$ & & & \\
\hline 153 & $\begin{array}{l}\text { P.D. } \\
\text { A.D. } \\
\text { A.G. } \\
\text { P.G. } \\
\text { Total }\end{array}$ & $\begin{array}{r}3,780 \\
2,980 \\
2,860 \\
3,990 \\
13,625\end{array}$ & $\begin{array}{r}3,790 \\
2,910 \\
2,840 \\
3,840 \\
13,380\end{array}$ & $\begin{array}{l}+0,010 \\
-0,070 \\
-0,020 \\
-0,150 \\
-0,245\end{array}$ & & & \\
\hline
\end{tabular}


TABLEAU 2

\begin{tabular}{|c|c|c|c|c|c|c|c|}
\hline $\begin{array}{c}\text { Vache } \\
\mathrm{N}^{\circ}\end{array}$ & Quartier & $\begin{array}{l}\text { Lait total } \\
\text { produit sous } \\
\text { influence de } \\
\mathrm{NaCl} 0,9 \%\end{array}$ & $\begin{array}{l}\text { Lait total } \\
\text { produit sous } \\
\text { influence de } \\
\text { la PHE } 5 \mathrm{mg}\end{array}$ & $\begin{array}{c}\text { Effet de } \\
\text { la PHE } \\
\text { sur le lait } \\
\text { total }\end{array}$ & $\begin{array}{c}\text { Lait total } \\
\text { produit sous } \\
\text { influence de } \\
\mathrm{NaCl} 0.9 \%\end{array}$ & $\begin{array}{l}\text { Lait total } \\
\text { produit sous } \\
\text { influence de } \\
\text { I'A }\end{array}$ & $\begin{array}{c}\text { Effet de } \\
\text { l'A } \\
\text { sur le lait } \\
\text { total }\end{array}$ \\
\hline 158 & $\begin{array}{l}\text { P.D. } \\
\text { A.D. } \\
\text { A.G. } \\
\text { P.G. } \\
\text { Total }\end{array}$ & $\begin{array}{r}\mathbf{8 , 3 7 0} \\
\mathbf{3}, 760 \\
1,010 \\
\mathbf{5 , 1 0 0} \\
\mathbf{1 8 , 2 5 0}\end{array}$ & $\begin{array}{r}7,580 \\
3,590 \\
1,060 \\
4,800 \\
\mathbf{1 7 , 0 4 0}\end{array}$ & $\begin{array}{l}-0,790^{*} \\
-0,170 \\
+0,050 \\
-0,300 \\
-\mathbf{1 , 2 1 0}\end{array}$ & $\begin{array}{r}5,680 \\
2,510 \\
0,600 \\
3,710 \\
12,500\end{array}$ & $\begin{array}{r}5,470 \\
2,580 \\
0,680 \\
3,790 \\
12,520\end{array}$ & $\begin{array}{l}-0,210 \\
+0,070 \\
+0,080 \\
+0,080 \\
-0,020\end{array}$ \\
\hline 181 & $\begin{array}{l}\text { P.D. } \\
\text { A.D. } \\
\text { A.G. } \\
\text { P.G. } \\
\text { Total }\end{array}$ & $\begin{array}{r}4,160 \\
2,830 \\
2,840 \\
3,300 \\
13,130 \\
\end{array}$ & $\begin{array}{r}3,620 \\
2,440 \\
2,750 \\
3,150 \\
11,960\end{array}$ & $\begin{array}{l}-0,540^{* * *} \\
-0,390^{* * *} \\
-0,090 \\
-0,150 \\
-1,170^{* *}\end{array}$ & & & \\
\hline 182 & $\begin{array}{l}\text { P.D. } \\
\text { A.D. } \\
\text { A.G. } \\
\text { P.G. } \\
\text { Total }\end{array}$ & $\begin{array}{r}4,690 \\
3,110 \\
3,220 \\
4,010 \\
15,050\end{array}$ & $\begin{array}{r}3,720 \\
2,680 \\
2,830 \\
3,420 \\
12,660\end{array}$ & $\begin{array}{l}-0,970^{* *} \\
-0,430^{*} \\
-0,390^{*} \\
-0,590^{* *} \\
-2,390^{* *}\end{array}$ & $\begin{array}{r}3,630 \\
2,530 \\
2,580 \\
3,300 \\
12,040\end{array}$ & $\begin{array}{r}3,160 \\
2,290 \\
2,370 \\
2,810 \\
10,630\end{array}$ & $\begin{array}{l}-0,470^{* *} \\
-0,240^{*} \\
-0,210 \\
-0,490^{* *} \\
-\mathbf{1}, \mathbf{4 1 0}\end{array}$ \\
\hline 186 & $\begin{array}{l}\text { P.D. } \\
\text { A.D. } \\
\text { A.G. } \\
\text { P.G. } \\
\text { Total }\end{array}$ & $\begin{array}{r}3,480 \\
2,600 \\
2,550 \\
3,450 \\
12,070 \\
\end{array}$ & $\begin{array}{r}2,990 \\
2,420 \\
2,370 \\
3,070 \\
10,850\end{array}$ & $\begin{array}{l}-0,490^{* *} \\
-0,180 \\
-0,180 \\
-0,380 \\
-1,220^{* *}\end{array}$ & & & \\
\hline 187 & $\begin{array}{l}\text { P.D. } \\
\text { A.D. } \\
\text { A.G. } \\
\text { P.G. } \\
\text { Total }\end{array}$ & $\begin{array}{r}3,123 \\
2,164 \\
2,031 \\
3,067 \\
10,385\end{array}$ & $\begin{array}{l}2,710 \\
1,945 \\
1,803 \\
2,658 \\
\mathbf{9 , 1 3 3}\end{array}$ & $\begin{array}{l}-0,413 \\
-0,219^{*} \\
-0,228 \\
-0,409^{*} \\
-1,252\end{array}$ & $\begin{array}{r}3,200 \\
2,040 \\
1,950 \\
3,100 \\
10,290\end{array}$ & $\begin{array}{l}2,940 \\
1,960 \\
1,880 \\
2,810 \\
\mathbf{9 , 5 9 0}\end{array}$ & $\begin{array}{l}-0,260^{*} \\
-0,080 \\
-0,070 \\
-0,290 \\
-0,700\end{array}$ \\
\hline 189 & $\begin{array}{l}\text { P.D. } \\
\text { A.D. } \\
\text { A.G. } \\
\text { P.G. } \\
\text { Total }\end{array}$ & $\begin{array}{r}2,770 \\
3,560 \\
3,440 \\
3,380 \\
13,160\end{array}$ & $\begin{array}{r}2,750 \\
2,890 \\
2,850 \\
3,090 \\
11,580\end{array}$ & $\begin{array}{l}-0,020 \\
-0,670^{* * *} \\
-0,590^{* * *} \\
-0,290^{* * *} \\
-1,580^{* * *}\end{array}$ & $\begin{array}{l}1,900 \\
2,360 \\
2,120 \\
2,400 \\
\mathbf{8 . 7 8 0}\end{array}$ & $\begin{array}{l}1,920 \\
2,380 \\
2,220 \\
2,440 \\
\mathbf{8 , 9 6 0}\end{array}$ & $\begin{array}{l}+0,020 \\
+0,020 \\
+0,100 \\
+0,040 \\
+\mathbf{0 , 1 8 0}\end{array}$ \\
\hline \multicolumn{2}{|c|}{ Moyenne générale } & 14,840 & 13,350 & $-1,490^{* * *}$ & 12,172 & 11,574 & -0.756 \\
\hline SEM & & 0,326 & 0,286 & 0,099 & 0,458 & 0,354 & 0,167 \\
\hline
\end{tabular}

Effet significatif au seuil de $0,05\left({ }^{*}\right) ; 0,01\left({ }^{*}\right)$ et $0,001\left({ }^{* * *}\right)$.

P.D. : postérieur droit ; A.D. : antérieur droit ; A.G. : antérieur gauche ; P.G. : postérieur gauche.

Avec l'adrénaline. - L'adrénaline $(0,3 \mathrm{mg})$ a également tendance à diminuer la production totale mais dans des proportions moindres $(-4,9 \% ; 11,57$ I sous A, 12,17 I sous $\mathrm{NaCl}$ ). Les animaux 135 et 182 sont néanmoins particulièrement sensibles au traitement et perdent respectivement $15 \%$ et $12 \%$ (tabl. 2).

Effets sur le volume de lait recueilli à l'égouttage.

$\mathrm{Ni}$ la phényléphrine ni l'adrénaline n'affectent significativement le volume de I'égouttage qui passe respectivement de $0,293 \mathrm{I}$ à $0,235 \mathrm{I}$ et de $0,430 \mathrm{I}$ à $0,360 \mathrm{I}$. 
Cette légère réduction de 58 à $70 \mathrm{ml}$ n'est pas suffisante pour expliquer la baisse de production laitière précédemment mentionnée. Celle-ci résulterait donc principalement d'une inhibition du réflexe d'éjection et ainsi d'une rétention importante de lait résiduel que les opérations mécaniques d'égouttage ne sont pas en mesure d'extraire de la lumière des acini mammaires.

\section{Effets sur le débit d'écoulement du lait lors de la traite mécanique.}

Injection de phényléphrine $(5 \mathrm{mg})$. - Le débit du lait observé en début de traite $(\mathrm{DO}=0,975 \mathrm{I} / \mathrm{min})$ n'est modifié ni par l'injection de $\mathrm{NaCl} 0,9 \%(\mathrm{D} 1=$ $0,962 \mathrm{l} / \mathrm{min}$ ), ni par celle de $5 \mathrm{mg}$ de phényléphrine (D2 $=0,961 \mathrm{l} / \mathrm{min}$ ) ce qui peut paraître surprenant puisque cette drogue est connue pour ses propriétés contractantes sur les fibres lisses du trayon (Bernabé et Peeters, 1980).

Quelques vaches présentent, certes, une faible diminution du débit mais celle-ci est compensée chez un nombre de sujets égal par un accroissement symétrique de ce même paramètre (tabl. 3).

TABLEAU 3

Effets des injections de $\mathrm{NaCl}$ et de PHE sur le débit lacté de chaque quartier.

\begin{tabular}{|c|c|c|c|c|c|c|}
\hline $\begin{array}{c}\text { Vache } \\
N^{\circ}\end{array}$ & Quartier & DO & D1 & $\mathrm{D} 1$ - D0 & $\mathrm{D} 2$ & $\mathrm{D} 2$ - D1 \\
\hline 120 & $\begin{array}{l}\text { P.D. } \\
\text { A.D. } \\
\text { A.G. } \\
\text { P.G. } \\
\text { Moyenne }\end{array}$ & $\begin{array}{l}2,608 \\
1,396 \\
1,335 \\
1,886 \\
\mathbf{1 , 8 0 6}\end{array}$ & $\begin{array}{l}2,899 \\
1,504 \\
1,184 \\
1,752 \\
1,835\end{array}$ & $\begin{array}{l}+0,291^{*} \\
+0,107 \\
-0,151 \\
-0,133 \\
+0,029\end{array}$ & $\begin{array}{l}2,814 \\
1,421 \\
1,333 \\
1,791 \\
1,840\end{array}$ & $\begin{array}{l}-0,084 \\
-0,083 \\
+0,148 \\
+0,039 \\
+\mathbf{0 , 0 0 5}\end{array}$ \\
\hline 135 & $\begin{array}{l}\text { P.D. } \\
\text { A.D. } \\
\text { A.G. } \\
\text { P.G. } \\
\text { Moyenne }\end{array}$ & $\begin{array}{l}1,159 \\
0,796 \\
0,631 \\
1,145 \\
\mathbf{0 , 9 3 3}\end{array}$ & $\begin{array}{l}1,106 \\
0,806 \\
0,639 \\
1,107 \\
\mathbf{0 , 9 1 5}\end{array}$ & $\begin{array}{l}-0,053 \\
+0,010 \\
+0,008 \\
-0,038 \\
-0,018 \\
\end{array}$ & $\begin{array}{l}1,291 \\
0,810 \\
0,760 \\
1,313 \\
1,044\end{array}$ & $\begin{array}{l}+0,186^{*} \\
+0,004 \\
+0,121^{*} \\
+0,206^{* *} \\
+0,129\end{array}$ \\
\hline 146 & $\begin{array}{l}\text { P.D. } \\
\text { A.D. } \\
\text { A.G. } \\
\text { P.G. } \\
\text { Moyenne }\end{array}$ & $\begin{array}{l}1,551 \\
1,156 \\
1,250 \\
1,530 \\
1,372\end{array}$ & $\begin{array}{l}1,337 \\
1,099 \\
1,212 \\
1,284 \\
1,233\end{array}$ & $\begin{array}{l}-0,214^{* * *} \\
-0,058 \\
-0,036 \\
-0,246^{* * *} \\
-\mathbf{0 , 1 3 9}\end{array}$ & $\begin{array}{l}1,383 \\
1,073 \\
1,280 \\
1,241 \\
\mathbf{1 , 2 4 4}\end{array}$ & $\begin{array}{l}+0,046 \\
-0,026 \\
+0,067 \\
-0,043 \\
+\mathbf{0 , 0 1 1}\end{array}$ \\
\hline 153 & $\begin{array}{c}\text { P.D. } \\
\text { A.D. } \\
\text { A.G. } \\
\text { P.G. } \\
\text { Moyenne }\end{array}$ & $\begin{array}{l}0,569 \\
0,421 \\
0,462 \\
0,557 \\
0,502\end{array}$ & $\begin{array}{l}0,531 \\
0,376 \\
0,441 \\
0,505 \\
\mathbf{0 . 4 6 3}\end{array}$ & $\begin{array}{l}-0,038 \\
-0,045^{*} \\
-0,022 \\
-0,052 \\
-0,039\end{array}$ & $\begin{array}{l}0,637 \\
0,423 \\
0,463 \\
0,562 \\
0,521\end{array}$ & $\begin{array}{l}+0,106 \\
+0,046^{* * *} \\
+0,022 \\
+0,057^{*} \\
+0,058\end{array}$ \\
\hline 158 & $\begin{array}{l}\text { P.D. } \\
\text { A.D. } \\
\text { A.G. } \\
\text { P.G. } \\
\text { Moyenne }\end{array}$ & $\begin{array}{l}1,399 \\
0,867 \\
0,133 \\
0,922 \\
\mathbf{0 , 8 3 0}\end{array}$ & $\begin{array}{l}\mathbf{1}, 289 \\
0,805 \\
0,153 \\
0,879 \\
\mathbf{0 , 7 8 1}\end{array}$ & $\begin{array}{l}-0,110 \\
-0,063 \\
+0,019 \\
-0,043 \\
-0,049\end{array}$ & $\begin{array}{l}0,972 \\
0,798 \\
0,158 \\
0,879 \\
\mathbf{0 , 7 0 2}\end{array}$ & $\begin{array}{c}-0,317^{*} \\
-0,007 \\
+0,055 \\
0 \\
-\mathbf{0 , 0 7 9}\end{array}$ \\
\hline
\end{tabular}


TABLEAU 3

\begin{tabular}{|c|c|c|c|c|c|c|}
\hline $\begin{array}{c}\text { Vache } \\
N^{\circ}\end{array}$ & Quartier & DO & D1 & D1 - D0 & D2 & $\mathrm{D} 2-\mathrm{D} 1$ \\
\hline 181 & $\begin{array}{l}\text { P.D. } \\
\text { A.D. } \\
\text { A.G. } \\
\text { P.G. } \\
\text { Moyenne }\end{array}$ & $\begin{array}{l}1,005 \\
0,602 \\
1,009 \\
0,965 \\
\mathbf{0 , 8 9 5}\end{array}$ & $\begin{array}{l}1,017 \\
0,675 \\
1,032 \\
1,036 \\
0,940\end{array}$ & $\begin{array}{l}+0,012 \\
+0,073^{*} \\
+0,022 \\
+0,072 \\
+0,045\end{array}$ & $\begin{array}{l}0,924 \\
0,634 \\
0,993 \\
0,958 \\
\mathbf{0 , 8 7 7}\end{array}$ & $\begin{array}{l}-0,093 \\
-0,041 \\
-0,039 \\
-0,078 \\
-0,063\end{array}$ \\
\hline 182 & $\begin{array}{l}\text { P.D. } \\
\text { A.D. } \\
\text { A.G. } \\
\text { P.G. } \\
\text { Moyenne }\end{array}$ & $\begin{array}{l}1,017 \\
0,832 \\
0,941 \\
0,971 \\
\mathbf{0 , 9 4 0}\end{array}$ & $\begin{array}{l}\mathbf{1}, 012 \\
0,859 \\
0,851 \\
0,925 \\
\mathbf{0 , 9 1 2}\end{array}$ & $\begin{array}{l}+0,005 \\
+0,026 \\
-0,090^{*} \\
-0,046 \\
-0,028\end{array}$ & $\begin{array}{l}1,024 \\
0,790 \\
0,852 \\
0,970 \\
\mathbf{0 , 9 0 9}\end{array}$ & $\begin{array}{l}+0,012 \\
-0,068 \\
+0,001 \\
+0,046 \\
-0,003\end{array}$ \\
\hline 186 & $\begin{array}{l}\text { P.D. } \\
\text { A.D. } \\
\text { A.G. } \\
\text { P.G. } \\
\text { Moyenne }\end{array}$ & $\begin{array}{l}0,981 \\
0,819 \\
0,968 \\
1,085 \\
\mathbf{0 , 9 6 3}\end{array}$ & $\begin{array}{l}1,016 \\
0,830 \\
1,029 \\
1,142 \\
\mathbf{1 , 0 0 4}\end{array}$ & $\begin{array}{l}+0,035 \\
+0,011 \\
+0,061 \\
+0,058 \\
+0,041\end{array}$ & $\begin{array}{l}1,045 \\
0,860 \\
0,970 \\
1,107 \\
\mathbf{0 , 9 9 6}\end{array}$ & $\begin{array}{l}+0,030 \\
+0,030 \\
-0,058 \\
-0,035 \\
-0,008\end{array}$ \\
\hline 187 & $\begin{array}{c}\text { P.D. } \\
\text { A.D. } \\
\text { A.G. } \\
\text { P.G. } \\
\text { Moyenne }\end{array}$ & $\begin{array}{l}0,782 \\
0,598 \\
0,627 \\
0,754 \\
\mathbf{0 , 6 9 0}\end{array}$ & $\begin{array}{l}0,752 \\
0,641 \\
0,610 \\
0,764 \\
\mathbf{0 , 6 9 1}\end{array}$ & $\begin{array}{l}-0,031 \\
+0,044 \\
-0,017 \\
+0,010 \\
+0,001\end{array}$ & $\begin{array}{l}0,692 \\
0,582 \\
0,588 \\
0,713 \\
\mathbf{0 , 6 4 4}\end{array}$ & $\begin{array}{l}-0,060 \\
-0,060 \\
-0,021 \\
-0,050 \\
-0,047\end{array}$ \\
\hline 189 & $\begin{array}{l}\text { P.D. } \\
\text { A.D. } \\
\text { A.G. } \\
\text { P.G. } \\
\text { Moyenne }\end{array}$ & $\begin{array}{l}0,838 \\
0,753 \\
0,792 \\
0,905 \\
\mathbf{0 , 8 2 2}\end{array}$ & $\begin{array}{l}0,834 \\
0,752 \\
0,851 \\
0,943 \\
\mathbf{0 , 8 4 5}\end{array}$ & $\begin{array}{l}-0,005 \\
-0,002 \\
+0,059 \\
+0,038 \\
+\mathbf{0 , 0 2 3}\end{array}$ & $\begin{array}{l}0,840 \\
0,727 \\
0,830 \\
0,925 \\
0,830\end{array}$ & $\begin{array}{l}+0,006 \\
+0,024 \\
-0,021 * * * \\
-0,018 \\
+0,015\end{array}$ \\
\hline \multicolumn{2}{|c|}{ Moyenne générale } & 0,975 & 0,962 & $-0,013$ & 0,961 & $+0,001$ \\
\hline SEM & & 0,044 & 0,044 & 0,007 & 0,044 & 0,007 \\
\hline
\end{tabular}

D0 : débit d'écoulement du lait avant toute injection ; D1 : débit d'écoulement du lait après injection de $\mathrm{NaCl} 0,9 \%$; D1-D0 : variation du débit ; D2 : débit d'écoulement du lait après injection de PHE 5 mg : D2-D1 : effet de la PHE.

Effet significatif au seuil de $0,05\left({ }^{*}\right) ; 0,01\left(^{* *}\right)$ et $0,001\left({ }^{* *}\right)$.

P.D., A.D., A.G., P.G. : voir tabl. 2.

Injection d'adrénaline $(0,3 \mathrm{mg})$. - Par contre, l'adrénaline, qui possède à la fois des pouvoirs $\alpha$-vasoconstricteur mammaire et $\beta$-relaxant sup la musculature du trayon, provoque une augmentation significative du débit lacté. Celui-ci passe de $0,977 \mathrm{l} / \mathrm{min}$ avant toute injection (DO) à $1,165 \mathrm{l} / \mathrm{min}$ après l'administration de l'adrénaline (D2). L'accroissement de $+8,7 \%$ par rapport au témoin $\mathrm{NaCl}$ (D1 = $1,072 \mathrm{l} / \mathrm{min}$ ) est significatif $(\mathrm{P}<0,5 \%)$. Parmi les 6 vaches de l'essai 4 sont responsables de l'effet et les animaux 120 et 182 ne semblent pas réagir à cette catécholamine (tabl. 4). 
TABLEAU 4

Effets des injections de $\mathrm{NaCl}$ et d'A sur le débit lacté de chaque quartier.

\begin{tabular}{|c|c|c|c|c|c|c|}
\hline Vache & Quartier & D0 & D1 & $\mathrm{D} 1-\mathrm{D} 0$ & D2 & $\mathrm{D} 2-\mathrm{D} 1$ \\
\hline 120 & $\begin{array}{l}\text { P.D. } \\
\text { A.D. } \\
\text { A.G. } \\
\text { P.G. } \\
\text { Moyenne }\end{array}$ & $\begin{array}{l}1,760 \\
1,291 \\
1,248 \\
1,664 \\
1,491\end{array}$ & $\begin{array}{l}2,067 \\
1,450 \\
1,530 \\
2,031 \\
1,770\end{array}$ & $\begin{array}{l}+0,307^{* *} \\
+0,159 \\
+0,282^{* * *} \\
+0,367^{*} \\
+\mathbf{0}, 279\end{array}$ & $\begin{array}{l}2,144 \\
1,545 \\
1,514 \\
1,828 \\
1,758\end{array}$ & $\begin{array}{l}+0,077 \\
+0,095 \\
-0,016 \\
-0,203 \\
-0,012\end{array}$ \\
\hline 135 & $\begin{array}{l}\text { P.D. } \\
\text { A.D. } \\
\text { A.G. } \\
\text { P.G. } \\
\text { Moyenne }\end{array}$ & $\begin{array}{l}1,128 \\
0,813 \\
0,546 \\
1,067 \\
0,888\end{array}$ & $\begin{array}{l}1,170 \\
0,827 \\
0,645 \\
1,117 \\
0,940\end{array}$ & $\begin{array}{l}+0,049 \\
+0,014 \\
+0,099 \\
+0,050 \\
+0,052\end{array}$ & $\begin{array}{l}1,462 \\
0,980 \\
0,780 \\
1,353 \\
1,144\end{array}$ & $\begin{array}{l}+0,292^{* *} \\
+0,153^{* *} \\
+0,135^{* *} \\
+0,236^{*} \\
+0,204^{*}\end{array}$ \\
\hline 158 & $\begin{array}{c}\text { P.D. } \\
\text { A.D. } \\
\text { P.G. } \\
\text { Moyenne }\end{array}$ & $\begin{array}{l}1,147 \\
0,915 \\
0,938 \\
1,000\end{array}$ & $\begin{array}{l}1,197 \\
1,072 \\
0,993 \\
1,087\end{array}$ & $\begin{array}{l}+0,050 \\
+0,157^{* *} \\
+0,055 \\
+0,087^{*}\end{array}$ & $\begin{array}{l}1,482 \\
1,033 \\
1,239 \\
1,251\end{array}$ & $\begin{array}{l}+0,285^{* * *} \\
-0,039 \\
+0,246^{* * *} \\
+0,164^{* * *}\end{array}$ \\
\hline 182 & $\begin{array}{l}\text { P.D. } \\
\text { A.D. } \\
\text { A.G. } \\
\text { P.G. } \\
\text { Moyenne }\end{array}$ & $\begin{array}{l}0,976 \\
1,053 \\
1,042 \\
1,026 \\
\mathbf{1 , 0 2 4}\end{array}$ & $\begin{array}{l}1,017 \\
1,093 \\
1,146 \\
1,067 \\
\mathbf{1 , 0 8 1}\end{array}$ & $\begin{array}{l}+0,041 \\
+0,040 \\
+0,104 \\
+0,041 \\
+0,057\end{array}$ & $\begin{array}{l}1,048 \\
1,168 \\
1,097 \\
1,056 \\
1,092\end{array}$ & $\begin{array}{l}+0,032 \\
+0,075 \\
-0,049 \\
-0,011 \\
+\mathbf{0 , 0 1 2}\end{array}$ \\
\hline 187 & $\begin{array}{l}\text { P.D. } \\
\text { A.D. } \\
\text { A.G. } \\
\text { P.G. } \\
\text { Moyenne }\end{array}$ & $\begin{array}{l}0,723 \\
0,667 \\
0,625 \\
0,735 \\
0,688\end{array}$ & $\begin{array}{l}0,698 \\
0,690 \\
0,643 \\
0,725 \\
0,689\end{array}$ & $\begin{array}{l}-0,025 \\
+0,023 \\
+0,018 \\
-0,010 \\
+0,001\end{array}$ & $\begin{array}{l}0,865 \\
0,750 \\
0,711 \\
0,814 \\
\mathbf{0 , 7 8 5}\end{array}$ & $\begin{array}{l}+0,167^{* * *} \\
+0,060 \\
+0,068^{*} \\
+0,089^{* *} \\
+0,096^{* * *}\end{array}$ \\
\hline 189 & $\begin{array}{l}\text { P.D. } \\
\text { A.D. } \\
\text { A.G. } \\
\text { P.G. } \\
\text { Moyenne }\end{array}$ & $\begin{array}{l}0,709 \\
0,815 \\
0,656 \\
0,890 \\
0,768\end{array}$ & $\begin{array}{l}0,809 \\
0,901 \\
0,776 \\
0,989 \\
0,869\end{array}$ & $\begin{array}{l}+0,100^{* *} \\
+0,086^{*} \\
+0,120^{* *} \\
+0,099^{* *} \\
+0,101^{* *}\end{array}$ & $\begin{array}{l}0,856 \\
1,065 \\
0,895 \\
1,014 \\
\mathbf{0 , 9 5 8}\end{array}$ & $\begin{array}{l}+0,047 \\
+0,164^{* *} \\
+0,119^{* * *} \\
+0,025 \\
+0,089^{* * *}\end{array}$ \\
\hline \multicolumn{2}{|c|}{ Moyenne générale } & 0.977 & 1,072 & $0,096^{* *}$ & 1,165 & $0,092^{* * *}$ \\
\hline SEM & & 0,031 & 0,057 & 0,013 & 0,051 & 0,013 \\
\hline
\end{tabular}

D0 : débit d'écoulement du lait avant toute injection ; D1 : débit d'écoulement du lait après injection de $\mathrm{NaCl} 0,9 \%$; D1-D0 : variation du débit ; D2 : débit d'écoulement du lait après injection de 0,3 mg d'A ; D2-D1 : effet d'A.

Effet significatif au seuil de $0,05(*) ; 0,01\left(^{* *}\right)$ et $0,001\left(^{* * *}\right)$

P.D., A.D., A.G., P.G. : voir tabl. 2.

\section{Discussion.}

L'adrénaline et la phényléphrine déterminent toutes les deux une baisse de production laitière mais n'affectent pas le volume du lait d'égouttage. - Ceci résulte probablement d'une rétention accrue de lait résiduel au niveau des acini 
mammaires, rétention consécutive à une vasoconstriction des artères mammaires (Dhondt et al., 1973) et à une perturbation de l'arrivée de l'ocytocine vers les cellules myoépithéliales. Les effets plus faibles de l'adrénaline semblent infirmer la présomption de $\beta$ récepteurs au niveau du myoépithélium émise par Vorherr (1971), tout au moins chez la vache.

Par contre, les actions de l'adrénaline et de la phényléphrine se distinguent très nettement quant à leur efficacité sur le débit d'écoulement du lait pendant la traite mécanique.

- Avec l'adrénaline, l'augmentation de l'écoulement du lait est similaire à celle observée précédemment avec l'isoprénaline, $\beta$-mimétique pur (Bernabé et Ricordel, 1985). Ceci confirme la prédominance de l'effet $\beta$-relaxant de l'adrénaline sur la musculature du trayon démontrée antérieurement par pléthysmographie (Bernabé et Peeters, 1980). L'hypotonicité, alors observée, facilite la déformation et donc l'ouverture du canal du trayon par la machine à traire,

- En opposition avec ce qui a été observé entre les traites sur des trayons n'ayant aucun contact avec la machine à traire (Bernabé et Peeters, 1980), la stimulation des $\alpha$ récepteurs par la phényléphrine ne freine pas l'écoulement du lait puisque les débits après phényléphrine ou $\mathrm{NaCl}$ restent identiques.

Ces effets différents des $\alpha$-mimétiques avant et pendant la traite suggèrent donc qu'un organe de ce type subissant des contraintes physiques de la part des manchons trayeurs (dont Balthazar et Scott, 1978, situent les valeurs les plus fortes au niveau du sphincter de l'organe), entre alors en état de contraction soutenue. Celle-ci serait suffisante pour rendre inefficace toute injection ultérieure $\mathrm{d}^{\prime} \alpha$ agoniste comme la phényléphrine. Delwich (1980) a d'ailleurs observé qu'en moyenne ces contractions s'établissent pendant la traite à une fréquence de 8 cycles par minute.

Cette hypothèse rend compte de l'augmentation du débit observé avec les $\beta$ mimétiques (adrénaline et isoprénaline); ceux-ci provoquent une relaxation des fibres musculaires, qui abaisse la résistance globale opposée par le sphincter aux sollicitations d'ouverture induite par la machine à traire.

Elle pourrait également rendre compte de la légère augmentation du débit, observée en fin de traite ou après injection de $\mathrm{NaCl}$, puisqu'il est plausible d'admettre un relâchement progressif du tonus musculaire au fur et à mesure que l'on s'éloigne du "stress" provoqué par la pose des gobelets.

Enfin, nos expériences minimisent l'influence du réseau vasculaire de la partie distale du trayon (hypothèse de congestion de Thiel et Mein, 1977) puisque 2 substances de vasomotricité opposée (adrénaline et isoprénaline) entraînent la même augmentation qualitative du débit lacté.

Reçu en septembre 1984. Accepté en octobre 1984.

Remerciements. - Nous tenons à remercier M. J. Labussière pour ses conseils, MM. F.A. de la Chevalerie, Y. Gauchet, C. Hiard, J. Lebail, E. Legendre, E. Lescouet pour leur assistance technique et Mme Annie Briand pour la dactylographie du manuscrit. 


\section{References}

BALTHAZAR J. M., SCOTT N. R., 1978 . Deformations of the dairy cow's teat by finite element analysis. Proc. int. Symp. on Machine Milking, 63-79. Cincinnati, Ohio. U.S.A.

BERNABÉ J., PEETERS G., 1980. Studies on the motility of smooth muscles of teats in lactating cows. J. Dairy Res., 47, 259-275.

BERNABÉ J., RICORDEL M. J., 1985. Modifications des conditions d'extraction du lait sous l'effet d'un $\beta$-mimétique (isoprénaline) administré par voie intrajugulaire pendant la traite mécanique de la vache. Repr. Nutr. Dévelop., 25, 61-74.

DHONDT G., HOUVENAGHEL A., PEETERS G., VERSCHOOTEN F., 1973. Influence of vasoactive hormones on mammary blood flow in lactating cows. Arch. int. Pharmacodyn. Ther., 204, 89.

DELWICHE M. J., SCOTT N. R., DROST C. J., 1980. Ultrasonic measurement of teat milk flow. Transact. ASAE, 746-752.

LABUSSIĖRE J., MARTINET J., 1964. Description de deux appareils permettant le contrôle automatique des débits de lait au cours de la traite à la machine. Premiers résultats obtenus chez la brebis. Ann. Zootech., 13, 199-212.

THIEL C. C., MEIN G. A., 1977. Machine milking. NIRD, Reading, England, p. 116.

VORHERR H., 1971. Catecholamine antagonism to oxytocin-induced milk ejection. Acta endocri. Scand., 154, 5-38. 